Physics

Physics Research Publications

Collective excitations of strongly interacting Fermi gases of atoms in a harmonic trap
Y. E. Kim
A. L. Zubarev

This paper is posted at Purdue e-Pubs.

http://docs.lib.purdue.edu/physics_articles/181 


\title{
Collective excitations of strongly interacting Fermi gases of atoms in a harmonic trap
}

\author{
Yeong E. Kim* and Alexander L. Zubarev ${ }^{\dagger}$ \\ Purdue Nuclear and Many-Body Theory Group (PNMBTG), Department of Physics, Purdue University, West Lafayette, \\ Indiana 47907, USA
}

(Received 28 February 2005; published 18 July 2005)

\begin{abstract}
The zero-temperature properties of a dilute two-component Fermi gas in the BCS to Bose-Einstein Condensation crossover are investigated. On the basis of a generalization of the Hylleraas-Undheim method, we construct rigorous upper bounds to the collective frequencies for the radial and the axial breathing mode of the Fermi gas under harmonic confinement in the framework of the hydrodynamic theory. The bounds are compared to experimental data for trapped vapors of ${ }^{6} \mathrm{Li}$ atoms.
\end{abstract}

DOI: 10.1103/PhysRevA.72.011603

PACS number(s): 03.75.Ss, 67.40.Db

The recently reported ultracold trapped Fermi gases with tunable atomic scattering lengths [1-19] in the vicinity of a Feshbach resonance stimulated a large number of theoretical investigations. In this Communication the dynamics of strongly interacting dilute Fermi gases (dilute in the sense that the range of interatomic potential is small compared with interparticle spacing) is investigated in the framework of the hydrodynamic theory [20-28].

Instead of using the scaling approximation of Refs. $[20,21,24,26]$, the polytropic approximation of Refs. [23,27], or the perturbative approximation of Ref. [25], we will construct a rigorous upper bounds to the collective frequencies.

Our starting point is the variational formulation of the Kohn-Sham time-dependent theory [29] for a dilute two-component Fermi gas in a trap potential $V_{\text {ext }}(\vec{r})$ $=(m / 2)\left[\omega_{\perp}^{2}\left(x^{2}+y^{2}\right)+\omega_{z}^{2} z^{2}\right]$,

$$
\delta \int d t\left\langle\Psi\left|i \hbar \partial_{t}-H\right| \Psi\right\rangle=0,
$$

where $|\Psi\rangle$ is a product of two Slater determinants, one for each internal state built up by the Kohn-Sham orbitals $\psi_{i}$, and $H=T+U$ is the Hamiltonian in the local-density approximation (LDA).

We consider the following two approximations:

(i) local transform $\psi_{i} \approx \phi_{i} \exp (i \chi)$, where $\phi_{i}$ and $\chi$ are real functions, and (ii) $\langle\phi|T| \phi\rangle \approx(1 / N) \int\left[t_{T F}(n)+t_{W}(n)\right] n(\vec{r}, t) d^{3} r$, where $n$ is the density, normalized to the total number of atoms $N, \int n d^{3} r=N,|\phi\rangle$ is the product of two Slater determinants built on $\phi_{i}$ alone, $t_{T F}(n)$ $=\left(3 \pi^{2}\right)^{2 / 3}\left[3 \hbar^{2} /(10 m)\right] n(\vec{r}, t)^{2 / 3}$ is the Thomas-Fermi (TF) kinetic-energy density, and $t_{W}(n)=\left[\hbar^{2} /(8 m)\right](\nabla n)^{2} / n$ is the von Weizsäcker kinetic-energy density [30].

The approximation (i) leads to the irrotational velocity field while the last term in (ii) leads to the quantum pressure.

Using the approximations (i) and (ii), we obtain from Eq. (1) $[20,21]$

\footnotetext{
*Email address: yekim@physics.purdue.edu

${ }^{\dagger}$ Email address: zubareva@ physics.purdue.edu
}

$$
i \hbar \frac{\partial \psi}{\partial t}=-\frac{\hbar^{2}}{2 m} \nabla^{2} \psi+V_{e x t} \psi+V_{x c} \psi
$$

where $\psi=\sqrt{n} \exp (i \chi)$ is the Madelung transform [31], $V_{x c}(\vec{r}, t)=(d[n \epsilon(n)] / d n)_{n=n(\vec{r}, t)}$ and $\epsilon(n)$ is the ground-state energy per particle of a uniform system. The only difference from equations holding for bosons $[32,33]$ is given by the density dependence of $\epsilon(n)$.

It is useful to rewrite Eq. (2) in a form

$$
\frac{\partial n}{\partial t}+\nabla(n \overrightarrow{\mathrm{v}})=0
$$

$$
\frac{\partial \overrightarrow{\mathrm{v}}}{\partial t}+\frac{1}{m} \nabla\left(V_{\text {ext }}+\frac{d[n \epsilon(n)]}{d n}+\frac{1}{2} m \mathrm{v}^{2}-\frac{\hbar^{2}}{2 m} \frac{1}{\sqrt{n}} \nabla^{2} \sqrt{n}\right)=0
$$

where $\vec{v}=(\hbar / m) \nabla \chi$ is the velocity field.

It was shown in Refs. [20,21] that for experimental conditions of Refs. $[14,16,17]$ the quantum pressure term in Eq. (4) [the last term in Eq. (4)] can be neglected. For the reminder of this Communication we will use this hydrodynamic approximation.

For the negative $S$-wave scattering length between the two fermionic species, $a<0$, in the low-density regime, $k_{F}|a|$ $\ll 1$, the ground-state energy per particle, $\epsilon(n)$, is well represented by an expansion in power of $k_{F}|a|$ [34],

$$
\begin{aligned}
\epsilon(n)= & 2 E_{F}\left[\frac{3}{10}-\frac{1}{3 \pi} k_{F}|a|+0.055661\left(k_{F}|a|\right)^{2}\right. \\
& \left.-0.00914\left(k_{F}|a|\right)^{3}+\cdots\right],
\end{aligned}
$$

where $E_{F}=\hbar^{2} k_{F}^{2} /(2 m)$ and $k_{F}=\left(3 \pi^{2} n\right)^{1 / 3}$. In the opposite regime, $a \rightarrow-\infty$ (the Bertsch many-body problem, quoted in Refs. [35]), $\epsilon(n)$ is proportional to that of the noninteracting Fermi gas

$$
\epsilon(n)=(1+\beta) \frac{3}{10} \frac{\hbar^{2} k_{F}^{2}}{m},
$$

where the universal parameter $\beta[10]$ is estimated to be $\beta=-0.56$ [36]. The universal limit $[10,36-40]$ is valid at 
least in the case where the width of the Feshbach resonance is large compared to the Fermi energy as in the cases of ${ }^{6} \mathrm{Li}$ and ${ }^{40} \mathrm{~K}$.

In the $a \rightarrow+0$ limit the system reduces to the dilute Bose gas of dimers [41]

$$
\epsilon(n)=E_{F}\left[-1 /\left(k_{F} a\right)^{2}+a_{m} k_{F} /(6 \pi)+\cdots,\right],
$$

where $a_{m}$ is the boson-boson scattering length, $a_{m} \approx 0.6 a$ [42].

A simple interpolation of the form $\epsilon(n) \approx E_{F} P\left(k_{F} a\right)$ with a smooth function $P(x)$ was considered in several papers. In Ref. [20] a [2/2] Padé approximant for the function $P(x)$ for the the case of negative $a$ has been proposed,

$$
P(x)=\frac{3}{5}-2 \frac{\delta_{1}|x|+\delta_{2} x^{2}}{1+\delta_{3}|x|+\delta_{4} x^{2}},
$$

where $\delta_{1}=0.106103, \quad \delta_{2}=0.187515, \quad \delta_{3}=2.29188, \quad \delta_{4}$ $=1.11616$. Equation (8) is constructed to reproduce the first four terms of the expansion (5) in the low-density regime and also to exactly reproduce results of the recent Monte Carlo calculations [36], $\beta=-0.56$, in the unitary limit, $k_{F} a \rightarrow-\infty$.

For the positive $a$ case (the interaction is strong enough to form bound molecules with energy $E_{m o l}$ ) we have considered in Ref. [21] a [2/2] Padé approximant

$$
P(x)=\frac{E_{m o l}}{2 E_{F}}+\frac{\alpha_{1} x+\alpha_{2} x^{2}}{1+\alpha_{3} x+\alpha_{4} x^{2}},
$$

where parameters $\alpha$ are fixed by two continuity conditions at large $x, 1 / x \rightarrow 0$, and by two continuity conditions at small $x$, $\alpha_{1}=0.0316621, \alpha_{2}=0.0111816, \alpha_{3}=0.200149$, and $\alpha_{4}$ $=0.0423545$.

In Ref. [43] a Padé approximation has been considered for the chemical potential. The authors of Ref. [27] have used a model for $P(x)$, interpolating the Monte Carlo results of Ref. [38] across the unitary limit and limiting behaviors for small $|x|$. We note here also the BCS mean-field calculations of Ref. [26]. form

The hydrodynamic equations after linearization take the

$$
\frac{\partial^{2}}{\partial t^{2}} \delta n+\frac{1}{m} \nabla\left[n \nabla\left(\frac{d^{2}[n \epsilon(n)]}{d n^{2}} \delta n\right)\right]=0,
$$

where $\delta n(\vec{r}, t)$ is the change in the density profile with respect to the equilibrium configuration. If we consider oscillations with time dependence $\delta n \propto \exp (i \omega t)$, Eq. (10) can be reduced to a Hermitian equation [25]

$$
-\omega^{2}\left(\frac{d^{2}[n \epsilon(n)]}{d n^{2}}\right)^{-1} f=\frac{1}{m} \nabla(n \nabla f),
$$

where $f=\left(d^{2}[n \epsilon(n)] / d n^{2}\right) \delta n$, and the equilibrium density, $n$, is given by equation

$$
\mu=V_{\text {ext }}+\frac{d[n \epsilon(n)]}{d n},
$$

where $\mu$ is the chemical potential, in the region where $n(\vec{r})$ is positive and $n(\vec{r})=0$ outside this region.
The Hilleraas-Undheim method [44] for upper bounds to eigenenergies of excited states of atoms can be generalized to the case of Eq. (11). Defining the functional $I[\chi]$ by

$$
I[\chi]=-(1 / m) \int \chi \nabla(n \nabla \chi) d^{3} r / \int \chi\left(\frac{d^{2}[n \epsilon(n)]}{d n^{2}}\right)^{-1} \chi d^{3} r,
$$

we get $I[f]=\omega^{2}$, where $f$ is the solution of Eq. (11).

Let the sequence of eigenvalues of Eq. (11) be denoted by $\omega_{1}^{2} \leqslant \omega_{2}^{2} \leqslant \ldots$ and let $\chi_{1}, \chi_{2}, \ldots, \chi_{q}$ form a set of $q$ linearindependent functions. Introducing the function $\chi=\sum_{r=1}^{q} c_{r} \chi_{r}$, where $c_{1}, c_{2}, \ldots, c_{q}$ are $q$ variable parameters, we see that the functional $I$ is stationary if $\partial I / \partial c_{r}=0$. Then the values of the $q$ roots, $\widetilde{\omega}_{1}^{2} \leqslant \widetilde{\omega}_{2}^{2} \leqslant \widetilde{\omega}_{3}^{2} \leqslant \cdots \leqslant \widetilde{\omega}_{q}^{2}$, of the secular equations

$\operatorname{det}\left[-\frac{1}{m} \int \chi_{p} \nabla\left(n \nabla \chi_{s}\right) d^{3} r-\widetilde{\omega}^{2} \int \chi_{p}\left(\frac{d^{2}[n \epsilon(n)]}{d n^{2}}\right)^{-1} \chi_{s} d^{3} r\right]$

$=0$

provide upper bounds to the $q$ lowest eigenvalues of Eq. (11) $\widetilde{\omega}_{r}^{2} \geqslant \omega_{r}^{2}$ for $r=1,2, \ldots, q$. It follows that as extra terms are added to the expansion of $\chi$, a given root of the secular equation $\widetilde{\omega}_{r}$ is decreased. An elegant proof of the HylleraasUndheim theorem is contained in Ref. [45].

For the most interested case of $M=0$ modes, we can put in Eq. (13) $q=3, \chi_{1}=1, \chi_{2}=\left(x^{2}+y^{2}\right)$, and $\chi_{3}=z^{2}$, which give

$$
\frac{\omega_{ \pm}^{2}}{\omega_{\perp}^{2}}=\frac{\eta \pm \sqrt{\eta^{2}-8 \lambda^{2} \zeta(5 \zeta-9)}}{(5 \zeta-9)} \text {, }
$$

where $\eta=\left(3+4 \lambda^{2}\right) \zeta-\left(3+6 \lambda^{2}\right), \quad \zeta=I_{0} I_{4} / I_{2}^{2}, \quad I_{l}=\int \widetilde{x}^{l} n(\tilde{x}) d \tilde{x}, \tilde{x}$ $=\sqrt{x^{2}+y^{2}+\lambda^{2} z^{2}}, \lambda=\omega_{z} / \omega_{\perp}$, and \pm signs refer to the transverse and axial modes, respectively.

To calculate $\zeta$, we have used the following expansion:

$$
n(\vec{r}) \approx\left[1-\beta V_{e x t}(\vec{r})\right]^{1 /(2-p)} \sum_{i=0}^{l-1} c_{i}\left[V_{e x t}(\vec{r})\right]^{i},
$$

where parameters $\beta, p$, and $c_{i}$ are fixed by requiring that $n(\vec{r})$ must satisfy a variational principle $\delta \int n\left[V_{\text {ext }}+\epsilon(n)\right] d^{3} r=0$ with a subsidiary condition $\int n d^{3} r=N$.

Since our upper bounds, Eq. (14), give exact solutions for frequencies of the breathing modes for the polytropic equation of state, $\epsilon(n) \approx n^{\gamma}$, we expect them to be close to the hydrodynamic frequencies near unitary limit.

The hydrodynamic equation is expected to be applicable for describing the macroscopic excitations of the system up to energies of the order of the energy gap, $\Delta$, needed to break up a Cooper pair. But for the trapped gas, $\Delta$ is a function of $\vec{r}$ ( $\Delta$ decreasing when we go away from the center). It is natural to assume that the condition of the applicability of hydrodynamics to describe the macroscopic excitations of the system at $T=0$ is $[21,46]$

$$
\frac{\hbar \Omega}{\tilde{\Delta}} \ll 1,
$$

where $\Omega$ is the frequency of the macroscopic excitations and the mean energy gap is given by $\widetilde{\Delta}=\int n(\vec{r}) \Delta(\vec{r}) d \vec{r} / N$. To calculate $\widetilde{\Delta}$ we have used the results of Refs. $[47,48]$. The pre- 


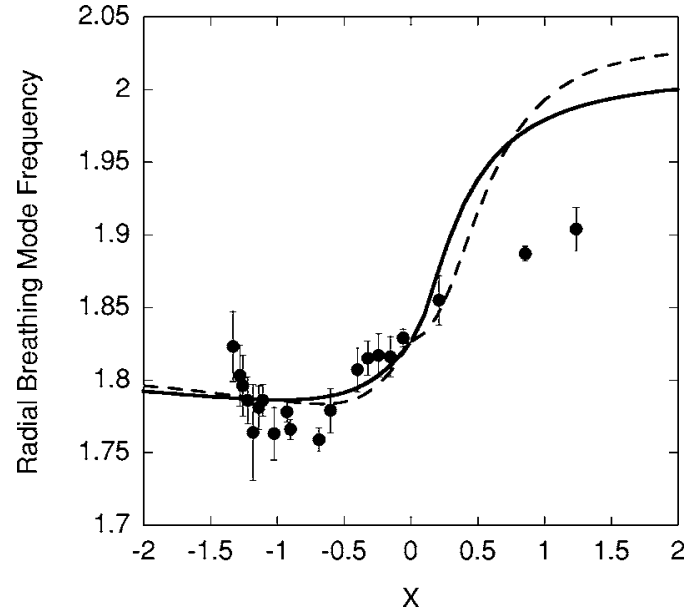

FIG. 1. Radial breathing mode frequency $\omega_{+}$in units of $\omega_{\perp}$ as a function of the dimensional parameter $X=\left(N^{1 / 6} a / a_{h o}\right)^{-1}$. In the unitary limit, $a \rightarrow-\infty$, one expects $\omega / \omega_{\perp}=\sqrt{10 / 3} \approx 1.826$. The solid and dashed lines represent the hydrodynamical upper bounds calculated using for the energy per particle the (2/2) Padé approximation of Refs. [20,21] and the parametrization of Ref. [27], respectively. The circular dots with error bars are the experimental results given by the Duke University group [17].

dictions of Eq. (14) for the radial breathing mode frequency of the cloud of ${ }^{6} \mathrm{Li}$ atoms are shown in Fig. 1. For $\epsilon(n)$ we have used two approximations, the Padé [2/2] approximation of Refs. [20,21] and the parametrization of Ref. [27]. A very small difference between these two approximations can be seen.

In Fig. 1, we have also compared the hydrodynamic predictions with experimental data [17]. We have used Ref. [49] to convert $a$ to the magnetic field $B$. Near the unitary limit there is a very good agreement with experimental data [17]. Away from this limit there is a qualitative agreement. We note here that two experimental results [14,17] and [16] (not shown in Fig. 1) for $\omega_{+}$are still about $10 \%$ in disagreement with each other, which is not fully understood yet. Reference [16] has found $\omega_{+} / \omega_{\perp} \approx 1.6$ at unitarity instead of the expected hydrodynamic value $\approx 1.8$. Since the collective frequencies are measured with high precision, this difference is significant. At $\left[N^{1 / 6} a / a_{h o}\right]^{-1}=-1.26$ the hydrodynamic upper bound begins to be below the sharp increased measured frequency that may confirm a breakdown of hydrodynamic theory [17]. In this region Ref. [17] observes also a rapid change in damping rate.

In Fig. 2, the calculated hydrodynamic upper bounds to the axial breathing mode frequency of the cloud of ${ }^{6} \mathrm{Li}$ is

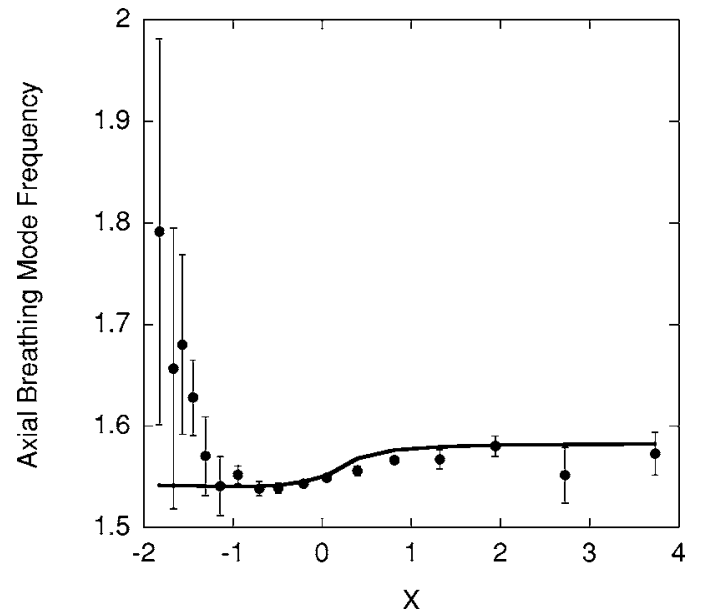

FIG. 2. Axial breathing mode frequency $\omega_{-}$in units of $\omega_{z}$ as a function of the dimensional parameter $X=\left(N^{1 / 6} a / a_{h o}\right)^{-1}$. The solid line represents the hydrodynamical upper bounds. The circular dots with error bars are the experimental results given by the Innsbruck group [16].

compared with experimental data [16] in the BCS to BoseEinstein condensation crossover region. Since the difference between approximations for $\epsilon(n)$ for this case is negligible, results with parametrization of Ref. [27] is not shown. A very good agreement between calculations and experimental data [16] for $\left[N^{1 / 6} a / a_{h o}\right]^{-1} \geqslant-1.14$ can be seen. However, at $\left[N^{1 / 6} a / a_{h o}\right]^{-1}=-1.31$ the hydrodynamic upper bound begins to be below the measured frequency that indicates, as in the radial mode case, breakdown of hydrodynamics, even though for the axial mode case the ratio between the collective energy and the gap energy, Eq. (16), is very small, $\hbar \omega_{-} / \widetilde{\Delta}$ $\approx 0.01$, at $\left[N^{1 / 6} a / a_{h o}\right]^{-1}=-1.31[50]$.

In summary, we have constructed the rigorous upper bounds to the collective frequencies of the Fermi gas under harmonic confinement in the framework of the hydrodynamic theory. The bounds are compared to experimental data on confined vapors of ${ }^{6} \mathrm{Li}$ atoms. It is shown that the hydrodynamic upper bound begins to be below the measured frequency at $\left[N^{1 / 6} a / a_{h o}\right]^{-1} \approx-1.3$, although the ratio between the collective energy and the gap energy, Eq. (16), for the axial mode is very small.

We thank J. Thomas for stimulating this work and for sharing with us the experimental results prior to publication. We also thank R. Grimm and M. Bartenstein for providing us with the updated experimental data.
[1] M. E. Gehm and J. E. Thomas, Am. Sci. 92, 238 (2004).

[2] C. A. Regal, M. Greiner, and D. S. Jin, Phys. Rev. Lett. 92, 040403 (2004).

[3] M. Greiner, C. A. Regal, and D. S. Jin, Nature (London) 426, 537 (2003).
[4] K. E. Strecker, G. B. Partridge, and R. G. Hulet, Phys. Rev. Lett. 91, 080406 (2003).

[5] J. Cubizolles, T. Bourdel, S. J. J. M. F. Kokkelmans, G. V. Shlyapnikov, and C. Salomon, Phys. Rev. Lett. 91, 240401 (2003). 
[6] S. Jochim, M. Bartenstein, A. Altmeyer, G. Hendl, C. Chin, J. H. Denschlag, and R. Grimm, Phys. Rev. Lett. 91, 240402 (2003).

[7] S. Jochim, M. Bartenstein, A. Altmeyer, G. Hendl, S. Riedl, C. Chin, J. H. Denschlag, and R. Grimm, Science 302, 2101 (2003).

[8] M. W. Zwierlein, C. A. Stan, C. H. Schunck, S. M. F. Raupach, S. Gupta, Z. Hadzibabic, and W. Ketterle, Phys. Rev. Lett. 91, 250401 (2003).

[9] C. A. Regal and D. S. Jin, Phys. Rev. Lett. 90, 230404 (2003).

[10] K. M. O’Hara, S. L. Hemmer, M. E. Gehm, S. R. Granade, and J. E. Thomas, Science 298, 2179 (2002).

[11] J. Kinast, A. Turlapov, J. E. Thomas, Q. Chen, J. Stajic, and K. Levin, Science 307, 1296 (2005).

[12] J. Kinast, A. Turlapov, and J. E. Thomas, Phys. Rev. Lett. 94, 170404 (2005); J. E. Thomas, J. Kinast, and A. Turlapov, cond-mat/0503620.

[13] M. Bartenstein, A. Altmeyer, S. Riedl, S. Jochim, C. Chin, J. Hecker Denschlag, and R. Grimm, Phys. Rev. Lett. 92, 120401 (2004).

[14] J. Kinast, S. L. Hemmer, M. E. Gehm, A. Turlapov, J. E. Thomas, Phys. Rev. Lett. 92, 150402 (2004).

[15] T. Bourdel, L. Khaykovich, J. Cubizolles, J. Zhang, F. Chevy, M. Teichmann, L. Tarruell, S. J. J. M. F. Kokkelmans, and C. Salomon, Phys. Rev. Lett. 93050401 (2004).

[16] M. Bartenstein, A. Altmeyer, S. Riedl, S. Jochim, C. Chin, J. Hecker Denschlag, and R. Grimm, Phys. Rev. Lett. 92, 203201 (2004); M. Bartenstein, A. Altmeyer, S. Riedl, S. Jochim, R. Geursen, C. Chin, J. Hecker Denschlag, and R. Grimm, cond-mat/0412712.

[17] J. Kinast, A. Turlapov, and J. E. Thomas, Phys. Rev. A 70, 051401(R) (2004).

[18] C. Chin, M. Bartenstein, A. Altmeyer, S. Riedl, S. Jochim, J. Hecker Denschlag, and R. Grimm, Science 305, 1128 (2004).

[19] M. W. Zwierlein, C. A. Stan, C. H. Schunck, S. M. F. Raupach, A. J. Kerman, and W. Ketterle, Phys. Rev. Lett. 92, 120403 (2004).

[20] Y. E. Kim and A. L. Zubarev, Phys. Lett. A 327, 397 (2004).

[21] Y. E. Kim and A. L. Zubarev, Phys. Rev. A 70, 033612 (2004); cond-mat/0408283.

[22] S. Stringari, Europhys. Lett. 65, 749 (2004).

[23] H. Heiselberg, Phys. Rev. Lett. 93, 040402 (2004).

[24] C. Menotti, P. Pedri, and S. Stringari, Phys. Rev. Lett. 89, 250402 (2002).

[25] A. Bulgac and G. F. Bertsch, Phys. Rev. Lett. 94, 070401 (2005).

[26] Hui Hu, A. Minguzzi, Xia-Ji Liu, and M. P. Tosi, Phys. Rev. Lett. 93, 190403 (2004).

[27] N. Manini and L. Salasnich, Phys. Rev. A 71, 033625 (2005).
[28] M. Cozzini and S. Stringari, Phys. Rev. Lett. 91, 070401 (2003).

[29] K. Burke and E. K. U. Gross, in Density Functionals edited by D. Joubert, Lecture Notes in Physics Vol. 500 (SpringerVerlag, Berlin, 1998), p. 123, and references therein.

[30] C. F. von Weizsäcker, Z. Phys. 96, 431 (1935).

[31] E. Madelung, Z. Phys. 40, 322 (1927).

[32] Y. E. Kim and A. L. Zubarev, Phys. Rev. A 67, 015602 (2003).

[33] Y. E. Kim and A. L. Zubarev, Phys. Rev. A 69, 023602 (2004).

[34] W. Lenz, Z. Phys. 56, 778 (1929); K. Huang and C. N. Yang, Phys. Rev. 105, 767 (1957); T. D. Lee and C. N. Yang, ibid. 105, 1119 (1957); V. N. Efimov and M. Ya. Amus'ya, Zh. Eksp. Teor. Fiz. 47, 581 (1964) [Sov. Phys. JETP 20, 388 (1965)].

[35] G. A. Baker, Jr., Int. J. Mod. Phys. B 15, 1314 (2001); Phys. Rev. C 60, 054311 (1999).

[36] J. Carlson, S.-Y. Chang, V. R. Pandharipande, and K. E. Schmidt, Phys. Rev. Lett. 91, 050401 (2003).

[37] R. Combescot, Phys. Rev. Lett. 91, 120401 (2003).

[38] G. E. Astrakharchik, J. Boronat, J. Casulleras, and S. Giorgini, Phys. Rev. Lett. 93, 200404 (2004).

[39] T. L. Ho and E. J. Mueller, Phys. Rev. Lett. 92, 160404 (2004); T. L. Ho, ibid. 92, 090402 (2004).

[40] G. M. Bruun, Phys. Rev. A 70, 053602 (2004).

[41] A. J. Leggett, in Modern Trends in the Theory of Condensed Matter, edited by A. Pekalski and R. Przystawa, Lecture Notes in Physics Vol. 115 (Springer-Verlag, Berlin, 1980) pp. 13-27; P. Nozières and S. Schmitt-Rink, J. Low Temp. Phys. 59, 195 (1985).

[42] D. S. Petrov, C. Salomon, and G. V. Shlyapnikov, Phys. Rev. Lett. 93, 090404 (2004).

[43] R. Combescot and X. Leyronas, Europhys. Lett. 68, 762 (2004).

[44] E. A. Hylleraas and B. Undheim, Z. Phys. 65, 759 (1930).

[45] R. G. Newton, Scattering Theory of Waves and Particles, 2nd ed. (Springer-Verlag, Berlin, 1982).

[46] R. Combescot and X. Leyronas, Phys. Rev. Lett. 93, 138901 (2004).

[47] L. P. Gorkov and T. K. Melik-Bakhudarov, Sov. Phys. JETP 13, 1018 (1961).

[48] S. Y. Chang, V. R. Pandharipande, J. Carlson, and K. E. Schmidt, Phys. Rev. A 70, 043602 (2004).

[49] M. Bartenstein, A. Altmeyer, S. Riedl, R. Geursen, S. Jochim, C. Chin, J. Hecker Denschlag, R. Grimm, A. Simoni, E. Tiesinga, C. J. Williams, and P. S. Julienne, Phys. Rev. Lett. 94, 103201 (2005).

[50] The measurements of the effective pairing gap [18] support a very small value of the ratio (16) for the axial mode case at $B=910 \mathrm{G}$ 